\title{
FOREWORD - ADVANCED OPTIMIZATION APPROACHES AND MODERN OR-APPLICATIONS
}

CODIT'14 conference or the International Conference on Control, Decision and Information Technologies has been held in Metz from 3 November to 5 November 2014. It has been the occasion to present 141 contributions selected from about 250 submissions (included the special sessions). This event has attracted participants from 34 countries. This special issue is devoted to CODIT'14 but the submission has been also extended to any high-quality paper non-presented at the conference. According to the journal standards, a new review process has been organized and applied to about 40 submitted papers to this special issue, which attempts to present some recent advances on optimisation and operations research and to publish original papers that contribute to the theory, the solution methods, and the applications related to these areas.

The scope of the 13 accepted papers is related to the advanced optimization/decision approaches and modern OR-applications (scheduling, vehicle routing problem, credit rating prediction, supply chain, underground transportation, sensor networks, multidimensional knapsack problem, graphs, portfolio selection, and dynamic facility layout problem). Such approaches play an important role in different domains and systems. The use of these practical tools can improve the quality, efficiency and performance of such systems. For this reason, the development of efficient optimisation algorithms has gained increasing interest in the recent decades. Research and development in these areas is growing rapidly due to the many successful applications of these approaches in very diverse problems.

In the first paper, Elalouf and Levner present an improved polynomial-time approximation algorithm belonging to the family of the fully polynomial time approximation schemes (FPTAS) for a group of scheduling problems, and to demonstrate it on a well-known problem: the scheduling problem with deadlines. Their algorithm provides a positive answer to a question posed more than three decades ago by Gens and Levner (1981): "Can an epsilon-approximation algorithm be found for the minimization version of the job-sequencingwith-deadlines problem running with the same complexity as the algorithms for the maximization form of the problem?".

In the second paper, Solano-Charris, Prins and Santos consider a bi-objective Vehicle Routing Problem, with uncertain demands and travelling times. Here, the objective is to minimize the worst total cost of traversed arcs and minimizing the maximum total unmet demand over all scenarios. To solve the problem, different hybrid multi-objective metaheuristics have been proposed and tested on different benchmarks.

In the third paper, Cheng, Chu, Chu and Xia investigate a bi-objective single-machine batch scheduling problem under Time-of-Use (TOU) electricity prices, with the aim of minimizing the makespan and the electricity costs. They propose new properties in order to reduce the search space of the Pareto optimal solutions. The paper presents the NP-hardness proof and a mixed integer linear programming model for the problem. Finally, 
they propose and test an exact $\varepsilon$-constraint method to identify its Pareto front and a fuzzy-logic-based approach to help decision makers in fixing the preferred solution.

In the fourth paper, Zhu, Chu, Yu and Sun study a single-machine scheduling problem with resource allocation and learning effect in the background of past-sequence-dependent (p-s-d) setup times. In their proposed model, the job processing times are dependent on learning effect and the amount of resource allocated, and the setup times are proportional to the length of the already processed jobs. They show that all the problems under study are polynomial while the complexity results are different. Moreover, they discuss some extensions and special cases of the studied problem.

In the fifth paper, Gangolf, Dochow, Schmidt and Tamisier consider the automated credit rating prediction (ACRP). Here, the aim is to predict the ratings of bonds without having to trust one rating agency (like Moody's, Fitch or S\&P). In this way, for a competitive ACRP algorithm, a worst-case guarantee concerning the misclassification error is offered. Moreover, several ACRP algorithms from the literature are compared according their competitiveness.

In the sixth paper, Ernez-Gahbiche, Hadjyoussef, Dogui and Jemai investigate the competitive and cooperative performances of a supply chain with two capacitated suppliers solicited by a customer who offers a new product procurement suggestion. Suppliers have the option to accept or reject the new product offer according to its profitability. In the competitive situation, they give the conditions that allow the principal supplier to select the best scheme. They show that the new product offer can be refused while it is accepted when suppliers cooperate. Finally, they present a profit allocation policy under which the collaboration is beneficial for the two suppliers.

In the seventh paper, Darties, Giroudeau, Konig and Simonin consider the makespan minimization coupledtasks problem in presence of compatibility constraints with a specified topology. In particular, they focus on stretched coupled-tasks, i.e. coupled-tasks having the same sub-tasks execution time and idle time duration. They study the complexity for several related problems and design some efficient polynomial-time approximation algorithms for an intractable scheduling problem according to some parameters.

In the eighth paper, Rathi and Balamohan consider the Sensor Mission Assignment (SMA) Problem in the context of Generalized Assignment Problem combined with decision making approach. They propose a twostage decision making approach to solve this problem under energy resource and environmental constraints. They simulate their method on a small sized wireless sensor network in order to prove its effectiveness.

In the ninth paper, Jiménez-Martin, Mateos and Fernàndez del Pozo review dominance measuring methods proposed in the literature. They report their possible drawbacks and compare their performance with other existing approaches. In particular, they illustrate dominance measuring methods in a real complex decisionmaking problem, which is based on the selection of cleaning services in a European underground transportation company.

In the tenth paper, Ferjani, Liouane and Borne propose a new variant of EAs called Logic Gate-based Evolutionary Algorithm (LGEA) to solve the Multidimensional Knapsack Problem (MKP). Their method introduces a new procedure for generating offspring using the logic gates' concept, which makes it possible to explore a major part of the search space. They test various MKP instances taken from the OR library and compare the provided results with those of other recently presented evolutionary methods.

In the eleventh paper, $\mathrm{Bi}$ and Meng investigate two optimal portfolio selection problems for an insurance company with proportional transaction costs: the maximization of the expected discounted utility of the total dividends, and the maximization of the insurer's expected utility of the terminal wealth. They propose 
bang-bang optimal strategies for these optimization problems. Using the homogeneous property, they reduce the dimensionality of the value function and give the numerical solutions to illustrate our results.

In the twelfth paper, Boumesbah and Chergui describe an exact method to generate the nondominated set of the minimum spanning tree problem with at least two criteria. The branching process of this method is done with respect to edges belonging to at least two cycles of a given graph, inducing a step of constructing linear constraints that progressively break cycles while respecting the connectivity of the resulting graph. As a consequence, the initial graph is portioned into subgraphs, each of which corresponds to a discrete multi-objective linear program allowing to finding the nondominated set of spanning trees. They evaluate the effectiveness of this method by testing it on random generated instances.

In the thirteenth paper, Hosseini and Seifbarghy propose an integrated approach for dynamic facility layout problem considering the material handling equipment (MHE). The aim is to minimize the fixed costs of MHE, the material handling cost (MHC) and the machine rearrangement costs (MRC). They propose an integrated multi-objective nonlinear mathematical programming model which is able to simultaneously select the MHE along with the arranging and re-arranging facilities. Considering the NP-hard nature, a novel Pareto-based metaheuristic algorithm called multi-objective water flow like algorithm (MOWFA) is developed to solve the problem. They discuss the parameter tuning of the algorithms utilizing Taguchi method and evaluate the performance of the proposed MOWFA compared to two well-known meta-heuristic algorithms (NSGA-II and NRGA).

Finally, the guest editors would like to address their warm thanks to all the reviewers for their great help in the evaluation of the submitted papers, the authors for their interesting contributions and Prof. Ridha Mahjoub, the editor-in-chief of RAIRO-Operations Research, for his help to the success of this project.

IMED KACEM, LCOMS, Université de Lorraine, UFR MIM, Ile du Saulcy, BP 30309, 57006 Metz cedex 1, France. imed.kacem@univ-lorraine.fr

ISOR, University of Graz, Graz, Austria.

HANS KeLLERER

hans.kellerer@uni-graz.at

ET PiERRE LAROChE. LCOMS, Université de Lorraine, UFR MIM, Ile du Saulcy, BP 30309, 57006 Metz cedex 1, France.

pierre.laroche@univ-lorraine.fr 